\title{
Blood Glucose Prediction Using Artificial Neural Networks Trained with the AIDA Diabetes Simulator: A Proof-of-Concept Pilot Study
}

\author{
Gavin Robertson, ${ }^{1}$ Eldon D. Lehmann, ${ }^{2}$ William Sandham, ${ }^{1,3}$ and David Hamilton ${ }^{4}$ \\ ${ }^{1}$ Bioengineering Department, University of Strathclyde, Wolfson Building, 106 Rottenrow, Glasgow G4 0NW, UK \\ ${ }^{2}$ Department of Imaging, CMRU, Imperial College of Science, Technology and Medicine, Royal Brompton Hospital, \\ Sydney Street, London SW3 6NP, UK \\ ${ }^{3}$ Scotsig, 40 Westbourne Gardens, Glasgow G12 9PF, UK \\ ${ }^{4}$ Ateeda Limited, CBC House, 24 Canning Street, Edinburgh EH3 8EG, UK \\ Correspondence should be addressed to William Sandham, w.sandham@scotsig.co.uk
}

Received 3 November 2010; Accepted 18 February 2011

Academic Editor: John Walsh

Copyright ( $\odot 2011$ Gavin Robertson et al. This is an open access article distributed under the Creative Commons Attribution License, which permits unrestricted use, distribution, and reproduction in any medium, provided the original work is properly cited.

\begin{abstract}
Diabetes mellitus is a major, and increasing, global problem. However, it has been shown that, through good management of blood glucose levels (BGLs), the associated and costly complications can be reduced significantly. In this pilot study, Elman recurrent artificial neural networks (ANNs) were used to make BGL predictions based on a history of BGLs, meal intake, and insulin injections. Twenty-eight datasets (from a single case scenario) were compiled from the freeware mathematical diabetes simulator, AIDA. It was found that the most accurate predictions were made during the nocturnal period of the 24 hour daily cycle. The accuracy of the nocturnal predictions was measured as the root mean square error over five test days $\left(\mathrm{RMSE}_{5}\right.$ day $)$ not used during ANN training. For BGL predictions of up to 1 hour a RMSE $\mathrm{R}_{5}$ ay of $( \pm \mathrm{SD}) 0.15 \pm 0.04 \mathrm{mmol} / \mathrm{L}$ was observed. For BGL predictions up to 10 hours, a RMSE 5 day of $( \pm S D) 0.14 \pm 0.16 \mathrm{mmol} / \mathrm{L}$ was observed. Future research will investigate a wider range of AIDA case scenarios, real-patient data, and data relating to other factors influencing BGLs. ANN paradigms based on real-time recurrent learning will also be explored to accommodate dynamic physiology in diabetes.
\end{abstract}

\section{Introduction}

The population with diabetes in 2000 was estimated to be 171 million worldwide. This figure is predicted to rise to 366 million by 2030 [1], which represents around $4.4 \%$ of the estimated worldwide population. However, it has been shown that, through good management of diabetes, its associated debilitating and costly complications can be reduced. The main complications can be divided into those that are short term, that is, hypoglycaemia and hyperglycaemia, and those that are long-term. The longer term complications can affect the vascular tree and the central nervous system as well as the kidneys and the eyes, resulting in cardiovascular disease, retinopathy, nephropathy, and neuropathy. For Type 1 (insulin-dependent) diabetics, the most common method for management is through with monitoring the blood glucose level (BGL) using fingerprick blood tests taken several times a day, and adjusting insulin doses based on these readings. For a dynamic, nonlinear, and complex condition such as diabetes this can be far from satisfactory. Factors such as insulin type and dose, diet, stress, exercise, illness, and pregnancy all have significant influences on the BGL. Management may be compromised through lack of data and, for some patients, an inability to interpret data adequately.

Devices such as continuous blood glucose meters (CGMs) and insulin pumps (IPs) may remove some of the burden of BGL management from the patient, through better monitoring and ease of supply of exogenous insulin. However, such devices work in an open-loop fashion, or pseudo closed-loop fashion requiring input and interpretation from the 
user. A system where these devices can work in a closedloop fashion, to maintain normoglycaemia with minimal intervention from the patient, may be the next step in diabetes management. However, such systems are currently only at the research and development stage, although initial clinical trials are promising [2].

To realise a closed-loop system for BGL control, that is, an artificial pancreas, at least three main components are required; a glucose monitor which is capable of registering autonomous BGL readings in real-time every few minutes, an autonomous insulin pump to supply insulin to a patient when needed, based on the BGL readings, and a control algorithm to predict the impact of insulin injections at some later time.

Control algorithms for BGL regulation tend to be grouped into two broad categories: the proportional-integralderivative (PID) controller and model predictive control (MPC) [3-6]. Essentially, the PID uses a classic feedback approach comparing the difference between the measured glucose level (blood or interstitial) and the expected glucose level, which is known as the proportional component. In addition, there is the integral component, which is the area under the curve between the measured glucose level and the expected glucose level, and the derivative component, which is the rate of change of the measured glucose level. From these components, proportional, integral, and derivative, the rate at which insulin is supplied to the body is adjusted. This is known as the insulin infusion rate (IIR), defined in the following equation, where $K_{P}, K_{I}$, and $K_{D}$ are the proportional, integral, and derivative weight terms, respectively, and $G$ and $G_{t}$ are the measured and expected (target) glucose levels, respectively [7]:

$$
\mathrm{IIR}=K_{P}\left(G-G_{t}\right)+K_{I} \int\left(G-G_{t}\right) d t+K_{D} \frac{d G}{d t}
$$

for MPC, the effects of carbohydrate intake and insulin injections on the BGL are captured in a glucoregulatory model. This can be in the form of a mathematical description of the various physiological processes of the body which can affect the BGLs. The glucoregulatory model may also be formulated as a "black box" approach, relying on pattern recognition from sets of training data. The "black box" approach is the method employed in the present research to model the AIDA physiological description, using artificial neural networks (ANNs).

ANNs represent a powerful modelling technique for pattern recognition, time series forecasting, and regression problems [8]. Their modelling ability relies on the fact that they do not require in-depth knowledge of the relationship between intrinsic variables for a particular problem, or the structure relating the variables required for mathematical modelling. Instead, an ANN is trained to recognise patterns in a dataset, and these patterns are effectively stored in the interconnected neuron weights of the ANN.

There is an extensive literature in the field of BGL prediction and control, using algorithms such as PID and MPC. Intersubject variability is one of the major challenges to be addressed in any closed-loop system which attempts to control the BGL of a patient, which is why ANNs may be a possible solution. An ANN can be trained on an individual's BGL; it can incorporate the factors specific to that person, without an in-depth knowledge of the individualspecific interactions which link them all. These factors can include, but are not limited to body mass index (BMI), age, gender, pregnancy, carbohydrate intake, exogenous insulin injections, exercise, and stress.

In the next section, the uses of ANNs for BGL prediction are reviewed.

\section{Artificial Neural Networks and Blood Glucose Prediction}

In this section, a brief review of existing ANN approaches to BGL prediction studies is presented. Performance measures quoted in the following studies give an indication of the prediction accuracies achieved by the various groups; however, due to differences in the data used and ANN architectures employed, direct comparisons with the findings of this paper may be difficult.

Sandham et al. [9] used an Elman recurrent ANN (designed and trained using MATLAB) with 95 neurons in the hidden layer to predict future BGL values using input parameters including insulin, diet, exercise, BGL, and other factors. A recurrent ANN was chosen for its superior performance in time-series prediction problems. Clinical data for training and evaluating the ANN were sourced from two patients taking regular BGL readings using fingerprick blood tests, together with diary entries recording information relating to the other input vectors. The development of the ANNs in this work was hindered by a lack of data, with the number of patients willing to cooperate cited as a problem. There was also concern over the accuracy of the meal data recorded by the patients.

Mougiakakou et al. [10] developed a simulation model for BGL prediction based on three compartmental models (CMs) describing short- and long-acting insulin effects on the glucose-insulin metabolism of a Type-1 diabetes patient, as well as a CM for glucose absorption from the gut. A recurrent $\mathrm{ANN}$, trained using a real-time recurrent learning algorithm, used the CMs as inputs to make a BGL prediction. Data from a Type-1 diabetes patient was used as the input to the system containing the CMs and the ANN. The data consisted of 69 days of measurements and contained information relating to BGLs (at breakfast, lunch, dinner, and bedtime), insulin injections, and food intake. The results were quoted as an RMSE between the ANN short-term prediction and data from the patient not used during ANN training. It was suggested that including additional patient information would enhance the model. Data relating to sex, age, other diseases, and years of diabetes, should result in a more patient-specific model. It was also expressed that data relating to physical exercise is an important parameter in BGL prediction, and its inclusion was considered important to improve accuracy.

Mougiakakou et al. [11] used a similar CM/ANN system in their work reported in [10]. This was used to predict BGLs for four children with Type-1 diabetes. The CMs (for insulin intake and glucose absorption from the gut) provided 
the inputs to an ANN, along with the most recent BGL. The ANNs used in this paper were a recurrent type (trained using a real-time recurrent learning algorithm) and a feed-forward variety (trained using a back-propagation algorithm). The BGL data used in this study were obtained from a CGM which provided a reading of interstitial glucose every 5 minutes measured over a period of days. Insulin and meal intake data were also recorded. The RMSE was used to compare the predicted BGL of the ANN with that of a day of patient data not used during training. ANNs were developed specifically for each patient. The results showed that the realtime recurrent network had similar performance to the feedforward type. However, the real-time recurrent network was preferred, due to its ability to adapt weights when a new input was supplied.

Zarkogianni et al. [12] used an ANN and CM developed in $[10,11]$ which have the ability to advise on insulin doses. This closed-loop system used a combined CM and ANN approach to make short-term BGL predictions, which were then fed into a nonlinear predictive controller capable of advising on insulin doses. Data were provided from a mathematical model, describing patients with Type-1 diabetes, with a sampling rate of 3 minutes. The results showed that the system can control BGLs with realistic meal intakes.

Pappada et al. [13] used ANNs generated from the NeuroSolutions software package to make BGL predictions over a 50- to 180-minute window. These were of a timelagged feed-forward type and were trained using the backpropagation algorithm. The data were acquired from 18 patients with Type-1 diabetes using a CGM to acquire blood glucose data over a period of 3-9 days for each patient, at a sampling rate of $1-5$ minutes. Training involved the use of datasets from 11-17 patients. In addition to this, an electronic diary was used by the patients to record insulin dosages, carbohydrate intake, hypo/hyperglycaemic symptoms, lifestyle (activities and events), and emotional states. The networks were tested using data acquired from the 18th patient not used in the training of the ANNs. Results were quoted as the mean absolute difference (MAD\%) between the ANN prediction and the output from the CGM. The MAD\% is defined in the following equation, where $\mathrm{NNet}_{\text {predict }, i}(t)$ is the ANN prediction, $\mathrm{CGM}_{\text {actual }, i}(t)$ is the target BGL from a CGM, and $N$ is the number of predictions:

$$
\begin{gathered}
\operatorname{AD}_{i}(t)=\frac{\left|\operatorname{NNet}_{\text {predict }, i}(t)-\mathrm{CGM}_{\text {actual }, i}(t)\right|}{\operatorname{CGM}_{\text {actual }, i}(t)} \times 100 \% \\
\operatorname{MAD} \%=\frac{\sum_{i=1}^{N} \operatorname{AD}_{i}(t)}{N}
\end{gathered}
$$

the results showed that predictions were more accurate for BGLs in the normoglycaemic and hyperglycaemic ranges than those in the hypoglycaemic range. One possible reason which was cited for this was that the dataset used for ANN training contained fewer examples of hypoglycaemic events than BGLs in other ranges. It was also shown that an increase in predictive length resulted in a decrease in predictive accuracy.
Pérez-Gandía et al. [14] used a feed-forward, fullyconnected ANN (multilayer perceptron) consisting of 3 layers. The first hidden layer had 10 neurons and the second had 5. The inputs to the ANN were the current BGL and $B G L$ readings up to 20 minutes prior to this reading. The data were sourced from 15 patients wearing continuous glucose monitors ( 9 wearing the Guardian REAL-Time from Medtronic-MiniMed and 6 wearing the FreeStyle Navigator from Abbott), with sampling frequencies ranging from 1 to 5 minutes. The training sets included both hypoglycaemic and hyperglycaemic events. Three ANNs were trained to predict 15,30 , and 45 minutes into the future, for a 24 -hour period. The results were quoted as an RMSE of the difference between the ANN prediction and patient data not used during the training of the ANN. For predictions of 15, 30, and 45 minutes, the RMSE error was $0.56,1.00$, and $1.5 \mathrm{mmol} / \mathrm{L}$, respectively. It was recognised that only using past CGM data as the input to the ANN limited the performance of the prediction system. It was suggested that including additional input data, such as meal intake and insulin dosage, could improve the ANN BGL prediction performance.

Curran et al. [15] presented a software solution using an ANN trained with the Levenberg-Marquardt algorithm to make BGL predictions based on expected insulin doses, carbohydrate intake, and exercise. The paper discusses a mobile phone-based application, INNSULIN, which takes data input from the user to advise on future BGLs, with the intention that insulin therapy and carbohydrate intake can be adjusted to maintain normoglycemia. The gathered data can be stored to generate a database of previous BGLs, insulin doses, carbohydrate intakes, and exercise, which can then be sent via a $3 \mathrm{G}$ connection to an endocrinologist, who can review the control of BGLs of a particular patient. Future work by the group will involve the development of the network to predict glucose levels 1 and 2 hours after the consumption of a meal, as well as predicting the amount of insulin necessary to control the BGL of the patient.

Hovorka [7] discusses the future of continuous glucose monitoring, and presents an interesting progression of the artificial pancreas. The author suggests an incremental development of the artificial pancreas as follows.

Generation 1. A system to terminate insulin infusion from an insulin pump at low BGLs to prevent hypoglycaemia. A closed-loop system for nocturnal control is also suggested, "when meal intake and exercise do not confound glucose control."

Generation 2. Glucose control around meal times with some user-interaction.

Generation 3. Closed-loop control around meals and periods of exercise.

Generation 4. Fully implantable systems or systems with dual control able to provide glucagon or other substances to prevent hypoglycaemic events.

Further reports of artificial neural network prototypes in diabetes care have also been described by other researchers [16-24]. 


\section{AIDA}

AIDA is a mathematical model and freeware diabetes simulator [25]. It models steady-state glucose-insulin interactions over a 24-hour period by describing the physiology of a person with Type-1 diabetes mellitus in terms of insulin sensitivity, glucose and insulin distribution, and the rate of gastric depletion. AIDA is the source of data for the ANNs developed in this paper. For more information on AIDA, readers should consult the website http://www.2aida.org/ or see the separate paper in this special issue [26].

The AIDA compartmental model takes into consideration a number of factors: carbohydrate intake (amount in grams and time), insulin (type, amount, and time), kidney function, and insulin sensitivity. Using these variables, a compartmental model of the various interactions within the body is described by a series of equations to build a picture of BGL fluctuations over a 24-hour period in time steps of 0.25 hours. It assumes a patient is unable to produce endogenous insulin, as is the case with Type- 1 diabetics.

AIDA is an acronym for automated insulin dosage advisor, a name originating from one of the historical goals of the project. Originally, it was hoped that it could be developed to make predictions of future BGLs for patients to maintain normoglycaemia by adjusting insulin doses. However, it became apparent that this model was not sufficiently accurate for individual BGL predictions or therapy planning. The authors make clear the limitations of this model. There are many warnings that it should not be used to make predictions for real-life patients' BGLs or to make changes to insulin regimens. Despite this limitation, there is still value in its capabilities as an educational tool for individuals with diabetes to manage their condition and, importantly for this project, in providing simulated data for initial ANN training, testing, and evaluation for a patient with diabetes.

The changes of renal threshold of glucose which occur with age and the effects of counterregulatory hormones (glucagon) at low BGLs have not, so far, been incorporated into the AIDA model. It also assumes that the effects of physical activity and stress remain relatively constant during the simulation period; these factors can have an impact on BGLs and need to be incorporated into future datasets which are used to train ANNs. In previous AIDA studies, it has also been found that parameters which were valid for one patient cannot be used to model all patients with a variety of conditions. The RMS deviation between predicted and observed BGLs increases as time progresses from the parameter-estimation period. It was also observed that the sensitivity parameters for insulin in the hepatic and peripheral regions were not necessarily accurate several days later [27], and it was noted that using the overall carbohydrate content of a meal, with no regard for parameters such as glycaemic index, was a simplification of the effect of a meal on BGL. The authors of the AIDA model also admit that current knowledge of the processes involved in the absorption of food from the gut is limited.

Despite these limitations, AIDA has proven to be a useful educational and research tool, and it has been applied by

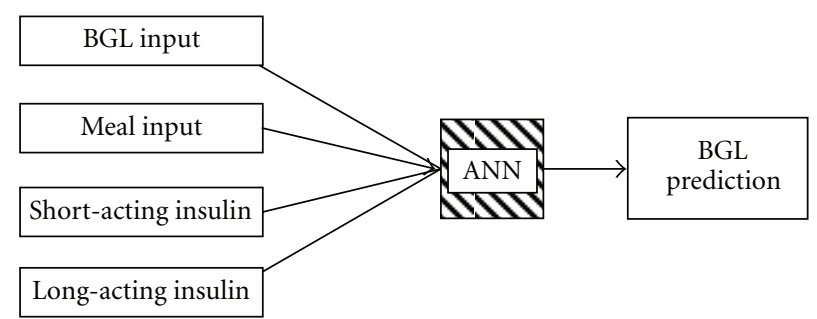

FIGURE 1: Schematic of inputs for short-term prediction ANN training.

a wide variety of research teams to harvest simulated BGL data for research purposes $[28,29]$.

\section{Preparing Blood Glucose Data from AIDA}

For short-term predictions (up to 1 hour), the ANNs were trained using all the available BGL data for a defined prediction period. The current time step was moved incrementally forwards through the prediction period, in time-steps of 0.25 hours (the resolution of the AIDA data). A trained ANN could, therefore, make BGL predictions for every time-step in the prediction period. This could be described as a moving window, with the current time-step at the centre (Table 1).

The input vector values precede the current time-step, with the target BGL defined at some future interval.

Twenty-eight days of data were produced from AIDA (single patient, Case Study 2) in which a day commenced at midday (12:00) and terminated at midday the following day. Each day contained the following events, which can be seen schematically in Figure 1:

BGL values, with a 15 minute frequency,

2 meals, timed to vary around 13:30 (Meal 1) and 19:00 (Meal 2) \pm 30 minutes,

2 short-acting insulin doses (these were timed to be administered 30 minutes before the meals \pm 15 minutes),

2 long-acting insulin doses (these were timed to be administered 30 minutes before Meal $1 \pm 15$ minutes and $1 \mathrm{hr}$ after Meal $2 \pm 15$ minutes). This could easily be modified to contain just one long-acting insulin dose for a 24-hour period.

For the majority of input vectors, the only non zero values were the BGLs, with the carbohydrate and insulin vectors, in most cases, being zero. The only periods where the insulin and carbohydrate vectors had a non zero value were around meal times, when insulin is required and carbohydrates are consumed (see Table 2).

For the long-term predictions, the same dataset was used as for the short-term predictions; however, it was presented differently to the ANN. Unlike for the short-term predictions, where the ANN was supplied with an input vector event every 15 minutes, the ANN was only given an input vector event if a meal had been consumed or an insulin injection had occurred. This resulted in the ANN 
TABLE 1: Incremental progression of the input vectors, current time-step, and BGL prediction used for ANN training.

\begin{tabular}{|c|c|c|c|c|c|c|c|c|c|}
\hline & & & Input & rectors & Current time step & & Prediction & & \\
\hline Time (hrs) & 18.25 & 18.50 & 18.75 & 19.00 & 19.25 & 19.50 & 19.75 & 20.00 & 20.25 \\
\hline $\mathrm{BGL}(\mathrm{mmol} / \mathrm{L})$ & 11.4 & 11.2 & 11.0 & 10.6 & 10.1 & 8.8 & 7.5 & 6.3 & 5.3 \\
\hline Meal (grams of carbohydrates) & 0 & 0 & 0 & 90 & 0 & 0 & 0 & 0 & 0 \\
\hline Short-acting insulin & 7 & 0 & 0 & 0 & 0 & 0 & 0 & 0 & 0 \\
\hline \multirow[t]{2}{*}{ Long-acting insulin } & 0 & 0 & 0 & 0 & 0 & 0 & 0 & 0 & 5 \\
\hline & & & & \multicolumn{2}{|c|}{ Input vectors } & Current time step & & Prediction & \\
\hline Time (hrs) & 18.25 & 18.50 & 18.75 & 19.00 & 19.25 & 19.50 & 19.75 & 20.00 & 20.25 \\
\hline $\mathrm{BGL}(\mathrm{mmol} / \mathrm{L})$ & 11.4 & 11.2 & 11.0 & 10.6 & 10.1 & 8.8 & 7.5 & 6.3 & 5.3 \\
\hline Meal (grams of carbohydrates) & 0 & 0 & 0 & 90 & 0 & 0 & 0 & 0 & 0 \\
\hline Short-acting insulin & 7 & 0 & 0 & 0 & 0 & 0 & 0 & 0 & 0 \\
\hline Long-acting insulin & 0 & 0 & 0 & 0 & 0 & 0 & 0 & 0 & 5 \\
\hline
\end{tabular}

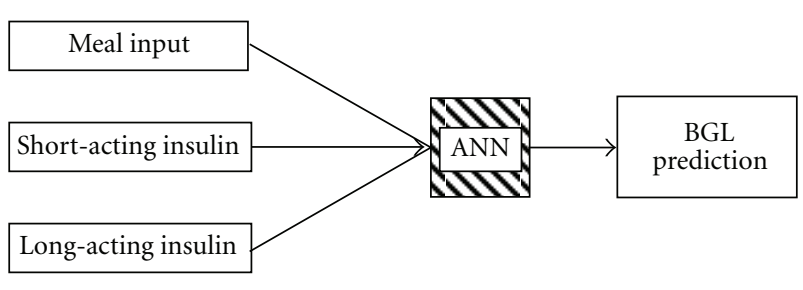

FIGURE 2: Schematic of inputs for long-term prediction ANN training.

only being given, for each day of data: 3 previous meal vectors, 3 short-acting insulin vectors, and 3 long-acting insulin vectors. A 24 -hour period began at midday and ran through to midday the following day. Each 24-hour period contained the following, which can be seen schematically in (Figure 2):

2 meals, timed to vary around 13:30 (Meal 1) and 19:00 (Meal 2) \pm 30 minutes, and the BGLs at these times;

2 short-acting insulin doses and the BGL at these events (these were timed to be administered 30 minutes before the meals \pm 15 minutes);

2 long-acting insulin doses and the BGL at the events (these were timed to be administered 30 minutes before Meal $1 \pm 15$ minutes, and $1 \mathrm{hr}$ after Meal $2 \pm 15$ minutes).

A sample of the data input vectors to the ANN is shown in Table 3. Note that time $0.00 \mathrm{hrs}$ represents midday and 12.00 hrs represents 24:00 (midnight).

The ANNs were trained to predict a single BGL value at $1,2,4,6,8$, or 10 hours after the last event supplied as an input, which was always a long-acting insulin injection, administered after Meal 2.

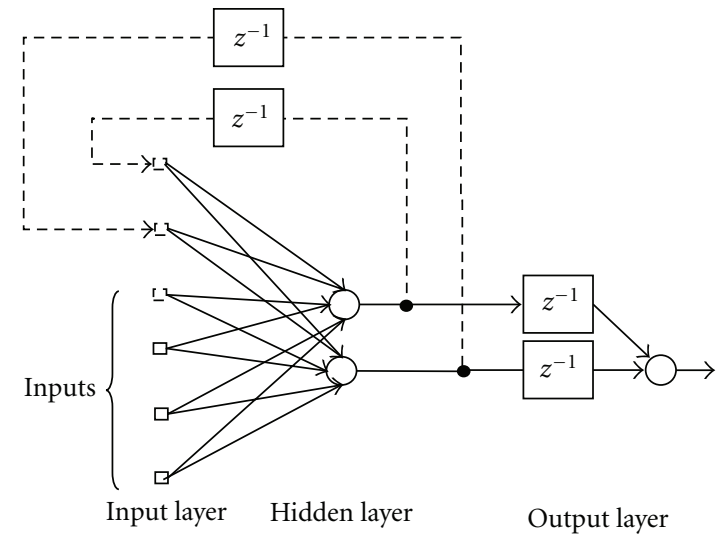

FIGURE 3: A fully connected recurrent neural network with 4 inputs, a hidden layer with 2 neurons, and 1 neuron in the output layer.

\section{ANN Architecture}

The ANNs were created using MATLAB version 7.8.0.347 (R2009a) and the Neural Networks Toolbox version 6.0.2 (R2009a). The ANNs developed in this project were based on the Elman recurrent ANN, which has been shown to have superior predictive abilities in time-series forecasting from feed-forward ANNs with no feedback loops. The Elman ANN is a two-layer ANN, with a feedback layer from the context layer to the input layer. This allows the ANN to have a "knowledge" of past events, which is a beneficial factor in predicting future events, such as in BGL prediction. The layout of the Elman ANN is shown in Figure 3 (adapted from [8]), which depicts a fully connected, recurrent Elman ANN with 4 inputs, 2 neurons in the hidden layer, and one output neuron. The 2 neurons in the hidden layer were found to be optimal through trial and error. The neurons of the hidden layer have a tansig activation function while the output neuron has a linear activation function. 
TABLE 2: A typical 2-event input vector supplied to the ANN for short-term predictions.

\begin{tabular}{|c|c|c|c|c|c|c|c|c|}
\hline \multicolumn{2}{|c|}{ Time (hours) } & \multicolumn{2}{|c|}{$\mathrm{BGL}(\mathrm{mmol} / \mathrm{L})$} & Meal (grams of carbohydrates) & \multicolumn{2}{|c|}{ Short-acting insulin } & \multicolumn{2}{|c|}{ Long-acting insulin } \\
\hline 12.25 & 12.5 & 4.253 & 3.984 & 0 & 0 & 0 & 0 & 0 \\
\hline
\end{tabular}

TABLE 3: Sample of the data used for training and testing of the ANNs for the long-term predictions (note that 0.00 hrs represents midday and 12.00 hrs represents midnight).

\begin{tabular}{|c|c|c|c|c|c|c|c|c|c|}
\hline \multirow{2}{*}{ Meal Input } & \multicolumn{3}{|c|}{ Time (hrs) } & \multicolumn{3}{|c|}{ Meal (grams of carbohydrates) } & \multicolumn{3}{|c|}{$\mathrm{BGL}(\mathrm{mmol} / \mathrm{L})$} \\
\hline & 1.25 & 3.75 & 7 & 56.7 & 0 & 75.6 & 12.384 & 4.996 & 5.710 \\
\hline Short-acting & \multicolumn{3}{|c|}{ Time (hrs) } & \multicolumn{3}{|c|}{ Short-acting insulin } & \multicolumn{3}{|c|}{$\mathrm{BGL}(\mathrm{mmol} / \mathrm{L})$} \\
\hline insulin input & 0.5 & 6.5 & 7.75 & 6 & 4 & 0 & 12.878 & 5.627 & 5.224 \\
\hline Long-acting & \multicolumn{3}{|c|}{ Time (hrs) } & \multicolumn{3}{|c|}{ Long-acting insulin } & \multicolumn{3}{|c|}{$\mathrm{BGL}(\mathrm{mmol} / \mathrm{L})$} \\
\hline insulin input & 0.5 & 6.5 & 7.75 & 4 & 0 & 6 & 12.878 & 5.627 & 5.224 \\
\hline
\end{tabular}

Training was performed using the Levenberg-Marquardt algorithm, defined in the following equation where $x_{k}$ and $x_{k+1}$ are measures of the performance function, $\mathbf{H}$ is a Hessian matrix, $\mu$ is a scalar, $\mathbf{I}$ is the identity matrix, and $\mathbf{g}$ is the gradient. This is regarded as a faster training algorithm than the standard back-propagation algorithm used historically in the Elman ANN, as it takes fewer epochs to find a minimum for the performance function:

$$
x_{k+1}=x_{k}-[\mathbf{H}-\mu \mathbf{I}] \mathbf{g}
$$

the training parameters for the Levenberg-Marquardt algorithm, used for the networks described in this project, are summarised below.

Weight Initialization. This was performed using the NguyenWidrow initialization function ("initnw" in MATLAB). The values are random to a certain degree, so any two networks will begin training with different neuron weights. Consequently, every network is unique, and networks trained on identical data will not necessarily give the same output.

Weight Updates. This was performed using the function called "trains", which is an incremental training algorithm presenting the network inputs and updating the weights with each time-step (epoch).

Data Allocation. This was performed using the function "dividerand," which is used to separate input and target vectors into three sets: training, validation, and testing. The data were split into the following ratio: training $60 \%$, validation $20 \%$, and testing $20 \%$. As the name of the function suggests, this is done in a random fashion.

Performance Measure. The criterion by which training success is assessed is the mean squared error (MSE). This takes the average of the sum squared of the errors, where the error is the difference between the network output and the target.
Training was terminated if $\mu$ reached $1 e 20$, or if the gradient $(\mathbf{g})$ reached $1 e-10$, or if a maximum number of training epochs (cycles) was obtained.

\section{Results}

The error in the prediction was calculated as the RMSE between the target (actual) BGL and the predicted BGL from the ANN over the prediction period, see (4), for 5 days of data not used during training. An average of the RMSE $\mathrm{E}_{\text {period }}$ over five test days was used to assess the performance of the ANN, RMSE 5 day:

$$
\begin{gathered}
\mathrm{RMSE}_{\text {period }}=\sqrt{\left(\mathrm{BGL}_{\text {actual }}-\mathrm{BGL}_{\text {predict }}\right)^{2}} \\
\mathrm{RMSE}_{5 \text { day }}=\overline{\sum_{i=1}^{5} \mathrm{RMSE}_{\text {period }, i}}
\end{gathered}
$$

an accurate BGL prediction was taken to be one which fell within the accuracy of glucose sensors, which was taken to be $\pm 1 \mathrm{mmol} / \mathrm{L}$. For short-term predictions, the prediction period ran from 18:00 until 06:30 the following day. The termination conditions for training were 100 epochs, a $\mu$ of $1 \mathrm{e} 20$, or a gradient of $1 e-10$.

From the graphs shown in Figures 4, 5, 6, and 7, it can be seen that the most accurate predictions of the ANNs were during the later portion of the night. The predictions are least accurate in the hours leading up to 24:00. If the predictions from 00:30 onwards are examined, then the average performance of the four individual ANNs, for prediction lengths $(15,30,45$, and 60 minutes $)$ is $\mathrm{RMSE}_{5}$ day $0.15 \pm 0.04 \mathrm{SD} \mathrm{mmol} / \mathrm{L}$ with an error $_{\max }$, as in the following equation, of $0.24 \mathrm{mmol} / \mathrm{L}$ at any one prediction time over the prediction period, for any network over the five test days:

$$
\text { error }_{\max }=\left|B \mathrm{BL}_{\text {actual }}-\mathrm{BGL}_{\text {predict }}\right|
$$

for longer-term predictions, the data were presented to the ANN in a different manner, as was mentioned earlier. Figures $8,9,10,11$, and 12 show the performance of the ANNs 


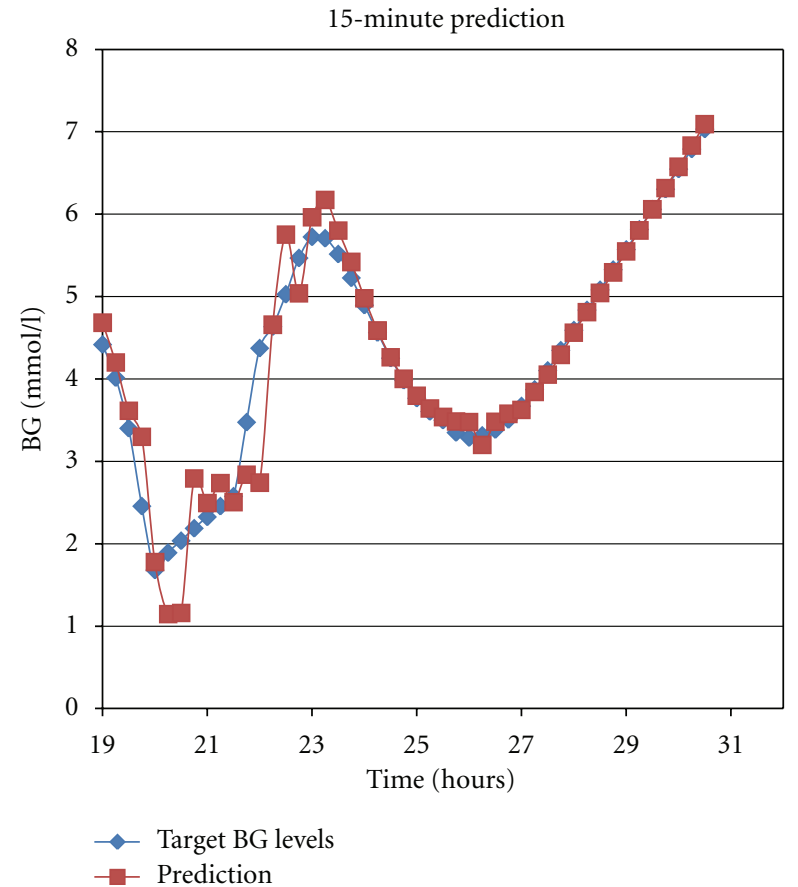

FIGURE 4: 15-minute prediction results from a day of data not used during AIDA training.

at predicting BGLs over time periods of $1,2,4,6,8$, and 10 hrs.

Two important points may be observed from Figure 8 to Figure 12.

Up to the 6-hour prediction, the difference between $\mathrm{BGL}_{\text {actual }}$ and $\mathrm{BGL}_{\text {predict }}$ (over the 5 test days) can be over $1 \mathrm{mmol} / \mathrm{L}$ (Figure 10).

The 8- and 10-hour predictions appear accurate: an average $\mathrm{RMSE}_{5 \text { day }}$ for the ANN for 8- and 10-hour predictions was $0.14 \pm 0.16 \mathrm{mmol} / \mathrm{L}$. The error max $_{\text {ax }}$ was $0.20 \mathrm{mmol} / \mathrm{L}$ for the 8 -hour prediction, while for the 10 -hour prediction the error max $_{\text {ax }}$ was $0.36 \mathrm{mmol} / \mathrm{L}$.

The accuracy of the predictions seems to improve as the prediction period increases away from the last input event. This could be explained by the diminishing influence on the BGL of the short/long-acting insulin and meal consumption as time progresses, making for a simpler modelling case, although further work is required to investigate this.

\section{Discussion}

The ANNs investigated in this paper were able to make the most accurate BGL predictions during the night. This is probably due to the reduced effect of meals and insulin injections during this period, although due to time constraints, a more rigorous investigation of this hypothesis could not be conducted. Networks with few hidden neurons appear to be able to make accurate BGL predictions of short-term nocturnal BGLs (up to 60 minutes) and long-term nocturnal blood glucose levels (8 and $10 \mathrm{hrs}$ ).

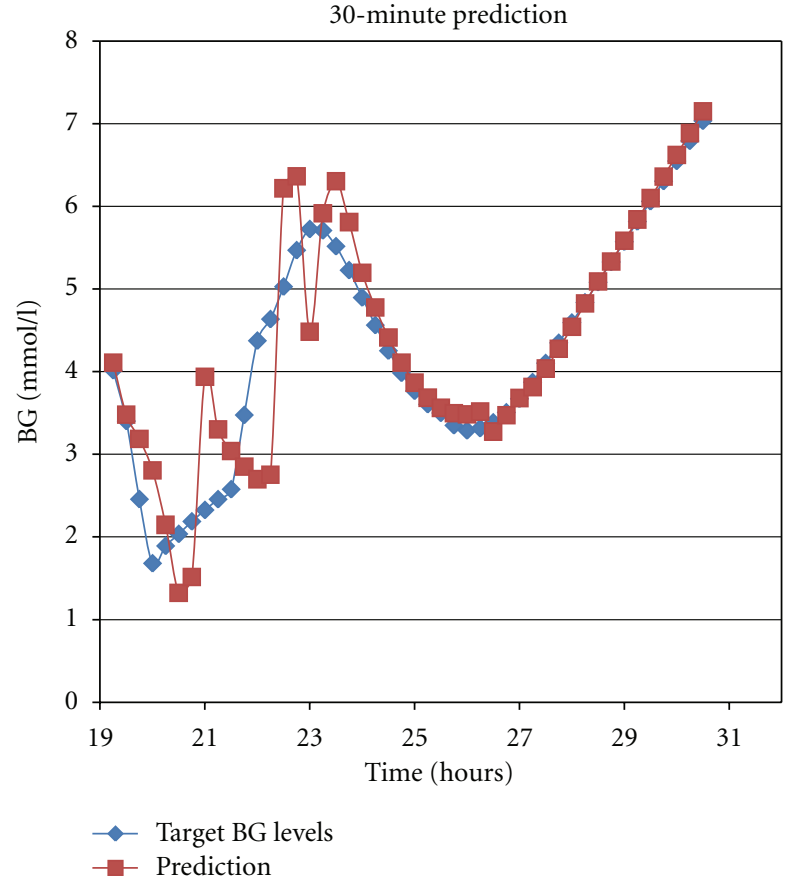

FIGURE 5: 30-minute prediction results from a day of data not used during AIDA training.

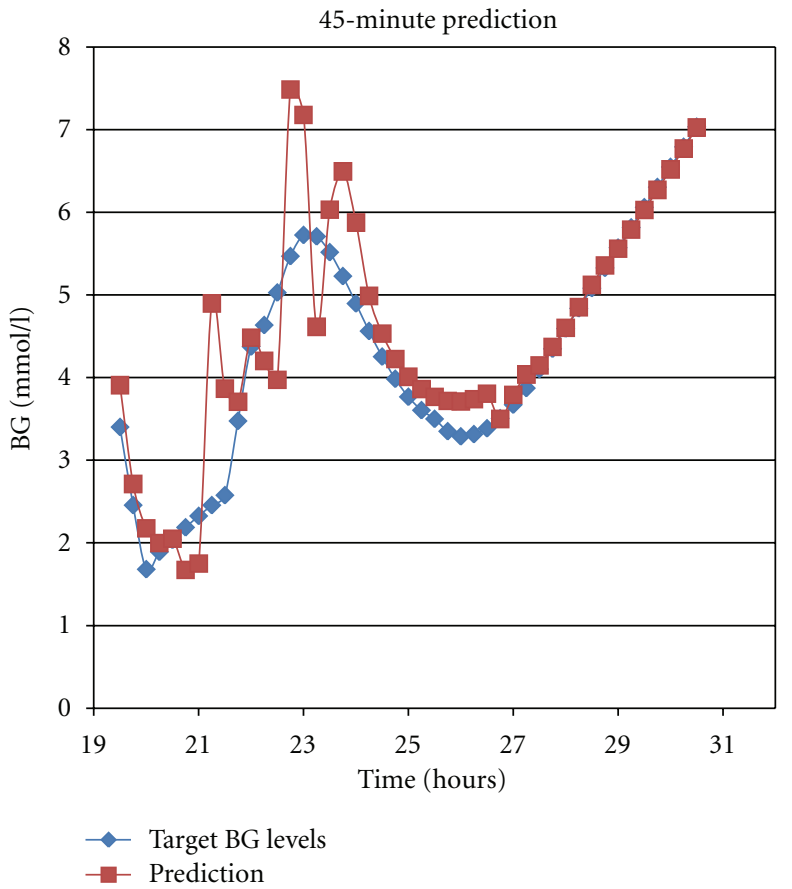

FIGURE 6: 45-minute prediction results from a day of data not used during AIDA training.

The results are similar to other work carried out [13, 14]; however, it should be noted that real-patient data was used in these papers, and that the prediction periods included daytime predictions. The BGLs found in the body are inherently unstable whereas those of the AIDA model are from a set of equations which are stable. There are 


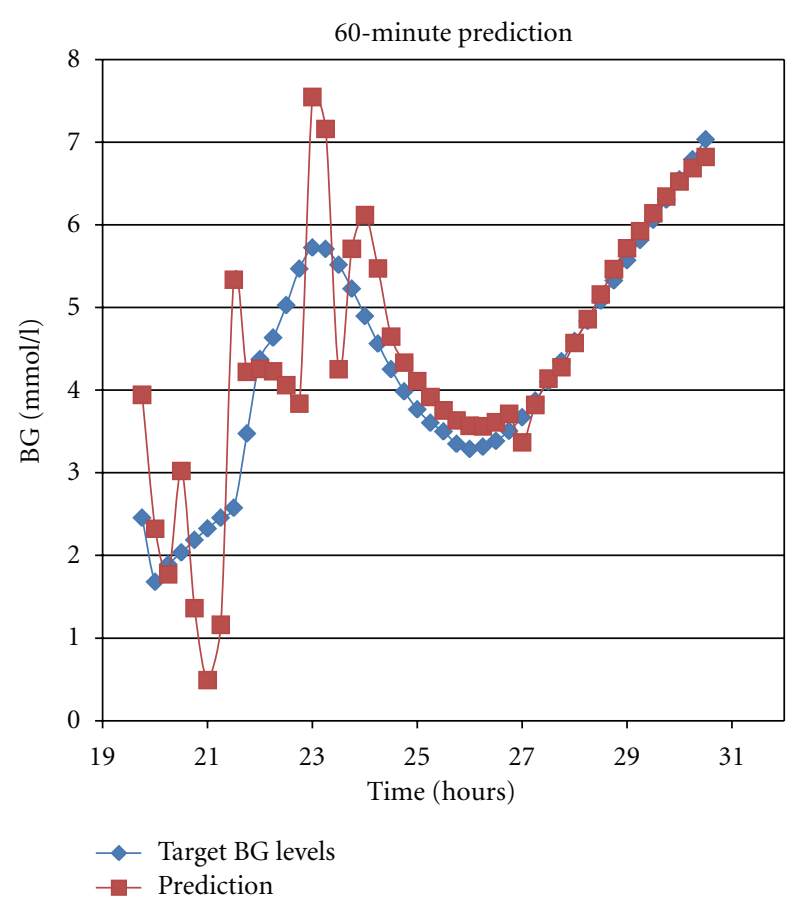

FIgURE 7: 60-minute prediction results from a day of data not used during AIDA training.

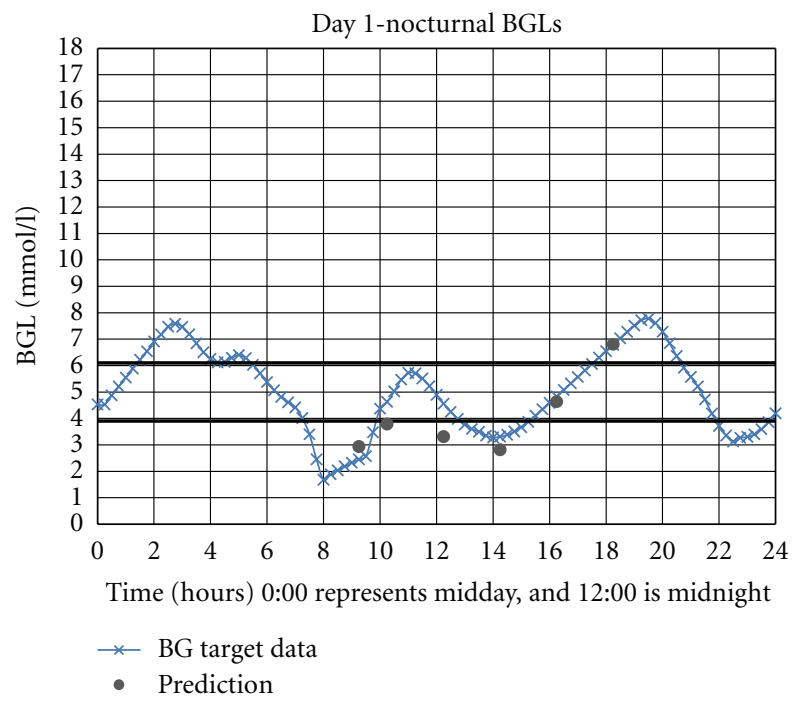

Figure 8: Predictions from trained ANNs on a test day which was not used during AIDA training. Each point represents a separate ANN, which was the best performing ANN over the 5 test days for a given prediction period.

factors during the nocturnal period which are not modelled by AIDA, and which may make predicting future BGLs more difficult. These include variations in glycemic index and protein content of meals, growth hormone and cortisol variations, sexual relations, restless sleep, excess hepatic gluconeogenesis created by the elevated glucose target for breakfast, or even an active dream life (the brain is the

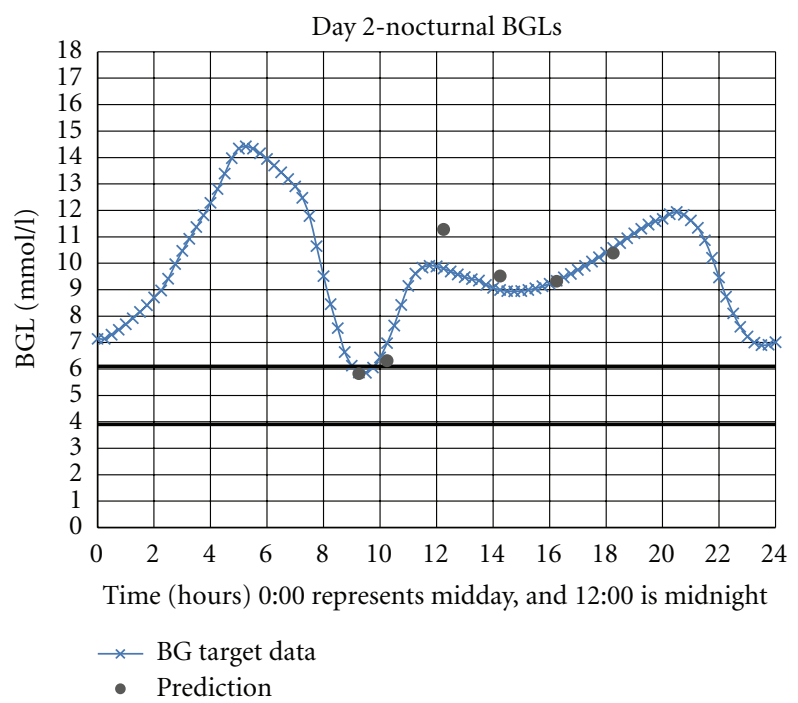

Figure 9: Predictions from trained ANNs on a test day which was not used during AIDA training. Each point represents a separate ANN, which was the best performing ANN over the 5 test days for a given prediction period.

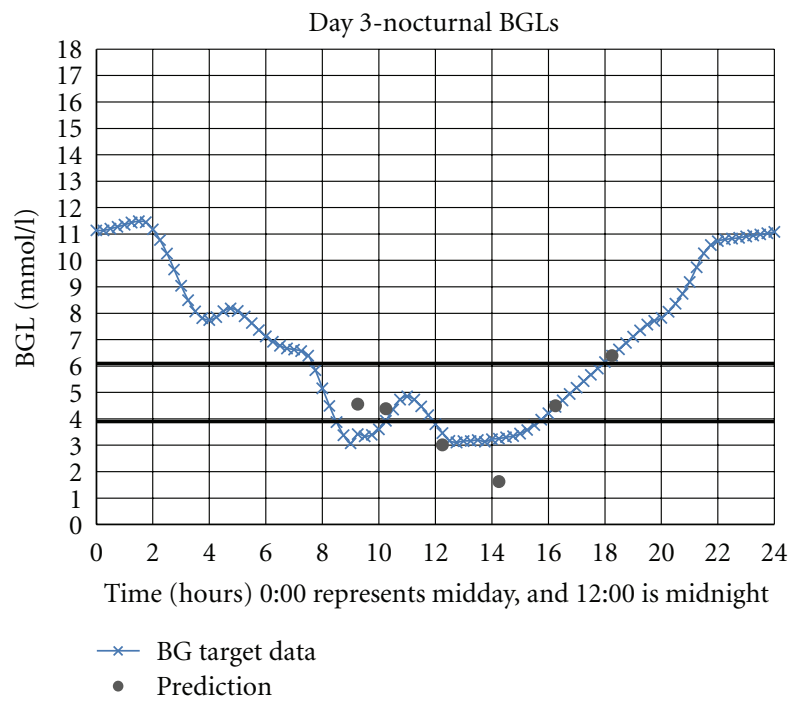

FIGURE 10: Predictions from trained ANNs on a test day which was not used during AIDA training. Each point represents a separate ANN, which was the best performing ANN over the 5 test days for a given prediction period.

major consumer of glucose during the night), but the night predictions of this project still seem valid. Control during the nocturnal period of the daily cycle may represent the first step towards a closed-loop artificial pancreas, as proposed for the first generation of artificial pancreases by Hovorka [7].

Although the results of this early work in progress are promising, there is much scope for improving the validity of the networks.

It is acknowledged that no test on real-patient data has yet been performed, and that the data of this project 


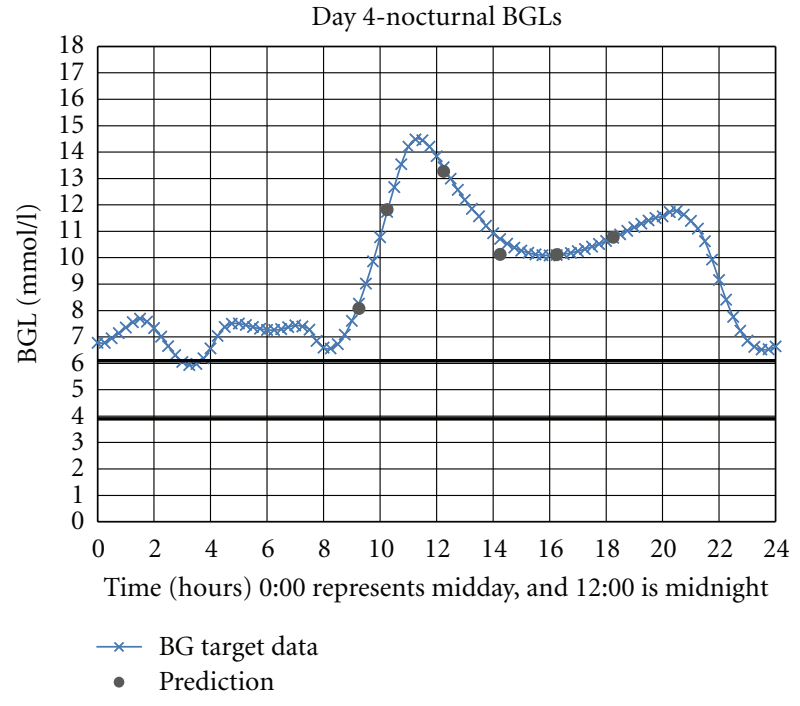

Figure 11: Predictions from trained ANNs on a test day which was not used during AIDA training. Each point represents a separate ANN, which was the best performing ANN over the 5 test days for a given prediction period.

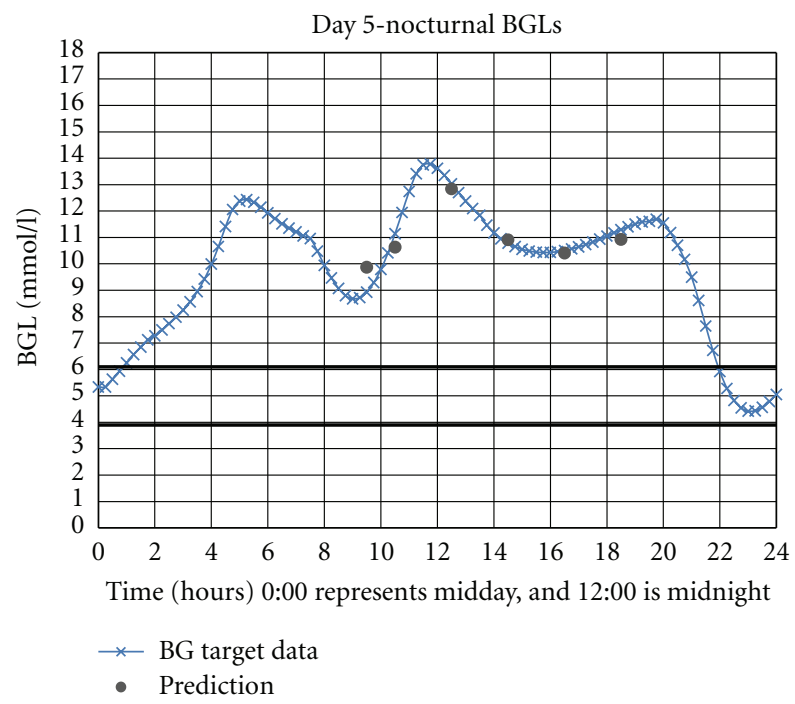

FIGURE 12: Predictions from trained ANNs on a test day which was not used during AIDA training. Each point represents a separate ANN, which was the best performing ANN over the 5 test days for a given prediction period.

was sourced from a limited mathematical model. This is a simplified case of a patient with Type-1 diabetes and does not include the effects of stress, exercise, illness, pregnancy, variations in glycemic index and protein content of meals, CGM detector noise, and so forth. All these variables will make for a more complicated situation and may reduce the overall accuracy of ANN predictions. However, using a model like AIDA does permit a large quantity of test BGL data to be harvested rapidly.
It is fully accepted that the simulation is only a starting point, but it is a useful way to embark on in silico testing. The purpose of this pilot study has been to prove the concept of this simulated data approach-but it is recognised that laboratory bench testing alone will not be sufficient-and if further in silico testing with a wider range of simulated patients is encouraging, then clinical testing with real-patient data will be warranted.

Using real-patient data, from a CGM with a sampling frequency similar to that of the AIDA sampling rate, would allow for investigation on the impact of glucose detector noise as well as the physiological responses of stress, exercise, illness, and so forth on the accuracy of prediction.

Using more than one patient will also be necessary to prove the validity of the prediction accuracy of the ANNs. This will allow for interindividual variability to be analysed. It should be stressed, however, that it was never the intention of this study to train a given ANN on more than one patient. This is with the belief that the trained ANN is specific to an individual's physiology, and that an ANN trained on more than one patient's BGL data would be inappropriate and have reduced accuracy. However, future work is needed to prove this.

Another issue with the AIDA model is the slow change in insulin sensitivities a patient can experience which can occur over the course of weeks and months. The parameters of the AIDA model, which are used to describe insulin sensitivities of the patients, are not necessarily valid a week after the data used to describe them was measured. The networks of this project are similar, in concept, to the AIDA model. Once the network has been trained, the weights, which essentially describe the physiological insulin/glucose interactions of a patient, are fixed. Over time, it would be expected that the accuracy of the network predictions would reduce as the patient's physiology changes. One way in which this issue could be addressed is by using a real-time recurrent learning network. This network differs from the Elman network, trained with the Levenberg-Marquardt algorithm, because it never stops training. Every time data is supplied to this network, small adjustments are made to the weights. In this way, it is able to adapt to slow changes in the data. Therefore, as the physiology of a patient changes with time, the network is able to incorporate the changes by adjusting the weights of its neurons. Such a network design was beyond the scope of this initial study.

\section{Conclusions}

ANNs, based on the Elman recurrent network and trained using the Levenberg-Marquardt algorithm, were designed, and were able to make accurate short- and long-term blood glucose predictions during the nocturnal period of the daily cycle.

For short-term predictions (15, 30, 45, and 60 minutes), ANNs made blood glucose predictions with $\mathrm{RMSE}_{5 \text { day }}$ of $0.15 \pm 0.04 \mathrm{SD} \mathrm{mmol} / \mathrm{L}$, and an error $\max$ of $0.27 \mathrm{mmol} / \mathrm{L}$. For long-term predictions ( 8 and $10 \mathrm{hrs}$ ), ANNs, with a $\mathrm{RMSE}_{5 \text { day }}$ predictions of $0.14 \pm 0.16 \mathrm{SD} \mathrm{mmol} / \mathrm{L}$, were 
observed. The error $\max$ was $0.20 \mathrm{mmol} / \mathrm{L}$ for the 8 -hour prediction and $0.36 \mathrm{mmol} / \mathrm{L}$ for the 10 -hour prediction.

Future areas of investigation will include developing alternative network paradigms which are capable of continuous learning, such as the real-time recurrent network. Such a network could be used in conjunction with the network configuration of this project to compare their prediction accuracy over an extended time period. This would highlight the effects which a changing metabolism might have on network prediction accuracy over the course of weeks or months. Such a study might also incorporate a real patient trial, utilising the data-gathering ability of CGMs, and digital diary logs of meal intake, insulin injections, exercise, and stress which could be easily incorporated into ANN paradigms.

\section{References}

[1] S. Wild, G. Roglic, A. Green, R. Sicree, and H. King, "Global prevalence of diabetes: estimates for the year 2000 and projections for 2030," Diabetes Care, vol. 27, no. 5, pp. 1047$1053,2004$.

[2] H. R. Murphy, D. Elleri, J. M. Allen et al., "Closed-loop insulin delivery during pregnancy complicated by type 1 diabetes," Diabetes Care, vol. 34, no. 2, pp. 406-411, 2011.

[3] M. W. Percival, H. Zisser, L. Jovanovic, and F. J. Doyle 3rd, "Closed-loop control and advisory mode evaluation of an artificial pancreatic Beta cell: use of proportional-integralderivative equivalent model-based controllers," Journal of Diabetes Science and Technology, vol. 2, no. 4, pp. 636-644, 2008.

[4] B. Grosman, E. Dassau, H. C. Zisser, L. Jovanovic, and F. J. Doyle 3rd, "Zone model predictive control: a strategy to minimize hyper- and hypoglycemic events," Journal of Diabetes Science and Technology, vol. 4, no. 4, pp. 961-975, 2010.

[5] J. J. Cordingley, D. Vlasselaers, N. C. Dormand et al., "Intensive insulin therapy: enhanced Model Predictive Control algorithm versus standard care," Intensive Care Medicine, vol. 35, no. 1, pp. 123-128, 2009.

[6] M. E. Wilinska, E. S. Budiman, M. B. Taub et al., "Overnight closed-loop insulin delivery with model predictive control: assessment of hypoglycemia and hyperglycemia risk using simulation studies," Journal of Diabetes Science and Technology, vol. 3, no. 5, pp. 1109-1120, 2009.

[7] R. Hovorka, "The future of continuous glucose monitoring: closed loop," Current Diabetes Reviews, vol. 4, no. 3, pp. 269279, 2008.

[8] S. Haykin, Neural Networks and Learning Machines, Pearson Education Inc, Upper Saddle River, NJ, USA, 3rd edition, 2009.

[9] W. Sandham, D. Nikoletou, D. Hamilton, K. Patterson, A. Japp, and C. Macgregor, "Blood glucose prediction for diabetes therapy using a recurrent artificial neural network," in Proceedings of the 9th European Signal Processing Conference (EUSIPCO '98), vol. 11, pp. 673-676, 1998.

[10] S. G. Mougiakakou, K. Prountzou, and K. S. Nikita, "A real time simulation model of glucose-insulin metabolism for type 1 diabetes patients," in Proceedings of the 27th Annual International Conference of the Engineering in Medicine and Biology Society (IEEE-EMBS '05), vol. 1, pp. 298-301, September 2005.

[11] S. G. Mougiakakou, A. Prountzou, D. Iliopoulou, K. S. Nikita, A. Vazeou, and C. S. Bartsocas, "Neural network based glucose - Insulin metabolism models for children with type 1 diabetes," in Proceedings of the 28th Annual International Conference of the IEEE Engineering in Medicine and Biology Society (EMBS '06), pp. 3545-3548, September 2006.

[12] K. Zarkogianni, S. G. Mougiakakou, A. Prountzou, A. Vazeou, C. S. Bartsocas, and K. S. Nikita, "An insulin infusion advisory system for type 1 diabetes patients based on non-linear model predictive control methods," in Proceedings of the Annual International Conference of the IEEE Engineering in Medicine and Biology Society, pp. 5972-5975, 2007.

[13] S. M. Pappada, B. D. Cameron, and P. M. Rosman, "Development of a neural network for prediction of glucose concentration in type 1 diabetes patients," Journal of Diabetes Science and Technology, vol. 2, no. 5, pp. 792-801, 2008.

[14] C. Pérez-Gandía, A. Facchinetti, G. Sparacino et al., "Artificial neural network algorithm for online glucose prediction from continuous glucose monitoring," Diabetes Technology and Therapeutics, vol. 12, no. 1, pp. 81-88, 2010.

[15] K. Curran, E. Nichols, E. Xie, and R. Harper, "An intensive insulinotherapy mobile phone application built on artificial intelligence techniques," Journal of Diabetes Science and Technology, vol. 4, no. 1, pp. 209-220, 2010.

[16] Z. Trajanoski and P. Wach, "Neural predictive controller for insulin delivery using the subcutaneous route," IEEE Transactions on Biomedical Engineering, vol. 45, no. 9, pp. 1122-1134, 1998.

[17] V. Tresp, T. Briegel, and J. Moody, "Neural-network models for the blood glucose metabolism of a diabetic," IEEE Transactions on Neural Networks, vol. 10, no. 5, pp. 1204-1213, 1999.

[18] S. G. Mougiakakou and K. S. Nikita, "A neural network approach for insulin regime and dose adjustment in type 1 diabetes," Diabetes Technology and Therapeutics, vol. 2, no. 3, pp. 381-389, 2000.

[19] E. Otto, C. Semotok, J. Andrysek, and O. Basir, "An intelligent diabetes software prototype: predicting blood glucose levels and recommending regimen changes," Diabetes Technology and Therapeutics, vol. 2, no. 4, pp. 569-576, 2000.

[20] G. Gogou, N. Maglaveras, B. V. Ambrosiadou, D. Goulis, and C. Pappas, "A neural network approach in diabetes management by insulin administration," Journal of Medical Systems, vol. 25, no. 2, pp. 119-131, 2001.

[21] W. Wang, Z. Z. Bian, L.-F. Yan, and J . Su, "A novel individual blood glucose control model based on mixture of experts neural networks," in Proceedings of the International Symposium on Neural Networks (ISNN '04), F. Yin,, J. Wang, and C. Guo, Eds., Lecture Notes in Computer Science 3174, pp. 453-458, Springer, 2004.

[22] A. K. El-Jabali, "Neural network modeling and control of type 1 diabetes mellitus," Bioprocess and Biosystems Engineering, vol. 27, no. 2, pp. 75-79, 2005.

[23] C. W. Ting and C. Quek, "A novel blood glucose regulation using TSK-FCMAC: a fuzzy CMAC based on the zero-ordered TSK fuzzy inference scheme," IEEE Transactions on Neural Networks, vol. 20, no. 5, pp. 856-871, 2009.

[24] G. Baghdadi and A. M. Nasrabadi, "Controlling blood glucose levels in diabetics by neural network predictor," in Proceedings of the 29th Annual International Conference of IEEE-EMBS, Engineering in Medicine and Biology Society (EMBC '07), pp. 3216-3219, August 2007.

[25] E. D. Lehmann and T. Deutsch, "A physiological model of glucose-insulin interaction in type 1 diabetes mellitus," Journal of Biomedical Engineering, vol. 14, no. 3, pp. 235-242, 1992. 
[26] E. D. Lehmann, C. Tarín, J. Bondia, E. Teufel, and T. Deutsch, "Development of AIDA v4.3b diabetes simulator technical upgrade to support incorporation of lispro, aspart and glargine insulin analogues," Journal of Electrical and Computer Engineering, vol. 2011, Article ID 427196, 17 pages, 2011.

[27] E. D. Lehmann, I. Hermanyi, and T. Deutsch, "Retrospective validation of a physiological model of glucose-insulin interaction in type 1 diabetes mellitus," Medical Engineering and Physics, vol. 16, no. 3, pp. 193-202, 1994.

[28] E. D. Lehmann, "Research use of the AIDA www.2aida.org diabetes software simulation program: a review-Part 1. Decision support testing and neural network training," Diabetes Technology and Therapeutics, vol. 5, no. 3, pp. 425-438, 2003.

[29] E. D. Lehmann, "Research use of the AIDA www.2aida.org diabetes software simulation program: a review-Part 2. Generating simulated blood glucose data for prototype validation," Diabetes Technology and Therapeutics, vol. 5, no. 4, pp. 641-651, 2003. 

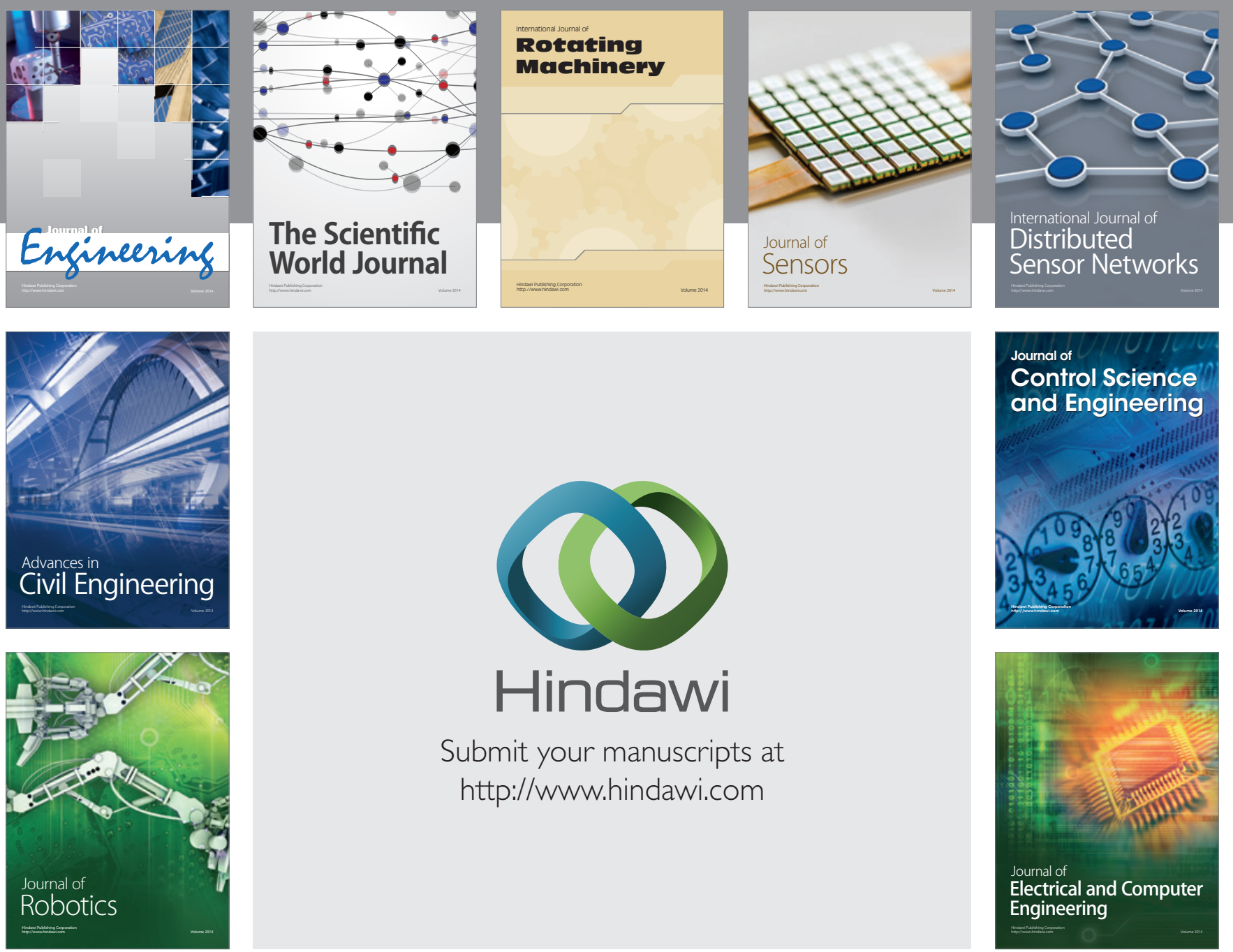

Submit your manuscripts at

http://www.hindawi.com
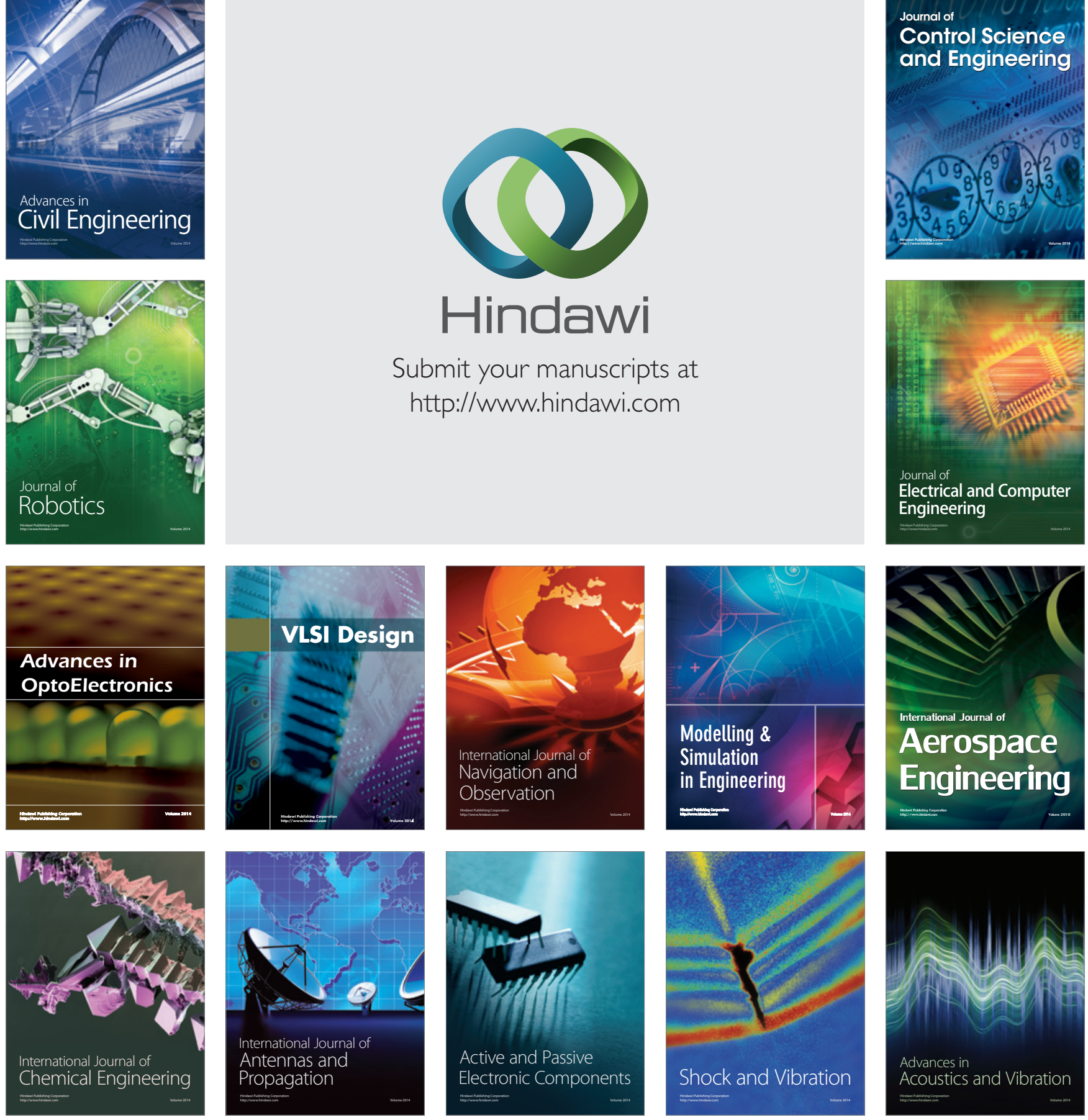